\title{
Evaluación de la aplicación de selladores en el marco asistencial de un programa público de salud bucodental
}

\author{
Navarro Montes I*, Peso de Ojeda L*, Herrera Ballesteros MA**, \\ González Sanz A***
}

RESUMEN

En el presente trabajo se evalúa la actividad " aplicación de selladores" llevada a cabo en el marco asistencial del Programa de Salud Bucodental infantil del SESCAM en dos Zonas Básicas de salud. Se analizan aspectos tales como eficacia, mantenimiento y grado de retención de los mismos, comparando nuestros resultados con los obtenidos por otros autores. Se concluye afirmando la idoneidad de la técnica en Atención Primaria así como la mayor posibilidad de pérdida del sellador a los 3 años de su aplicación y si el escolar reside en medio urbano, no encontrando, en nuestro estudio, relación entre pérdida de sellador y molar sellado.

Palabras clave: Programa de Salud Bucodental. selladores, supervivencia, retención.

\section{SUMMARY}

In this work, it is evaluated the activity "sealants application" carried out in the welfare framework of the program of the bucodental health childlike of the SESCAM in two Basic Zones of Health. Aspects are analyzed such as efficacy, mainteanceand degree of retention of the same, comparing our results whit them obtained by other authors. It is concluded afirming the smitability of the techniqur in primary attention, as well as the greater posibility of loss of the sealant to the trhee years of his application and if the student resides in the middle urban, not finding in our study relation among loss of sealant and molar sealed.

Key words: Program of Bucodental health, sealants, survival, retention.

Aceptado para publicación: octubre 2003.

* $\quad$ Odontólogo de Área. SESCAM. Ciudad Real.

** Higienista dental de Área. SESCAM. Ciudad Real.

*** Profesor Titular Dpto. Estomatología IV. Fac. Odontología. UCM.

Navarro Montes I, Peso de Ojeda L, Herrera Ballesteros MA, González Sanz A. Evaluación de la aplicación de selladores en el marco asistencial de un programa público de salud bucodental. Av. Odontoestomatol 2004; 20-1: 33-40.

\section{INTRODUCCIÓN}

Desde hace 10 años, en nuestra Área de Salud está implantado un Programa de Salud Bucodental infantil (PSBD) que oferta sus servicios en el ámbito de la Atención Primaria de Salud (APS) y está ubicado físi- camente en los Centros de Salud. En la actualidad, la cobertura alcanzada con red asistencial pública es del $90 \%$ de la población diana (P.D) a la que va dirigido el PSBD (escolares de 6-14 años de edad), no existiendo concierto con entidades privadas para esta atención odontológica. 
Entre las prestaciones ofertadas en el marco del PSBD , se encuentran la aplicación de selladores de fosas y fisuras en los primeros molares permanentes dado que ha quedado demostrado que aportan una protección contra la caries del $100 \%$ si permanecen íntegros ${ }^{1}$. La efectividad del sellador se incrementa si cuando éste se pierde total o parcialmente es repuesto mediante control periódico ${ }^{2}$.

Si la pérdida del sellador se produce principalmente en los 6 primeros meses ésto se debe a errores en la técnica de aplicación. Pasado ese tiempo, la pérdida del sellador puede deberse al desgaste oclusal o a un fracaso marginal. La posible duración de los selladores se calcula entre 10 y 15 años tras la colocación de los mismos por un único especialista ${ }^{3}$.

Por otra parte, hay pruebas de que el número de bacterias descienden en la fisura cuando está sellada, por lo que la progresión de una caries incipiente se podría detener ${ }^{4}$. Esto sucede, por un lado, porque el proceso de grabado destruye las bacterias y por otro, porque el sellador hace que los microorganismos "se mueran de hambre" evitando que les lleguen nutrientes ${ }^{5}$.

Aunque el higienista dental desarrolla una labor fundamental durante el proceso de colocación del sellador, la planificación y decisión sobre la colocación de los mismos depende exclusivamente del odontólogo, pudiendo aplicar el higienista el sellador, siendo esta labor coste-efectiva, ahorrándose tiempo y dejando al dentista para otros menesteres ${ }^{6}$.

\section{OBJETIVOS}

1) Valorar la implantación de selladores de fosas y fisuras en nuestra P.D. así como la eficacia y mantenimiento de los mismos, en primeros molares permanentes.

2) Evaluar el grado de retención alcanzado en relación con el molar tratado.

\section{MATERIAL Y METODO}

Durante el periodo comprendido entre los años 1994 a 1999 ( ambos incluidos) hemos estudiado un total de 2749 escolares de 9 a 10 años de edad. De ellos 1372 eran niños y 1377 niñas. Al inicio del estudio cursaban $4^{\circ}$ de primaria en 15 colegios ( 12 públicos y 3 concertados ) de 6 poblaciones de la provincia de Ciudad Real. 2195 escolares se sometieron voluntariamente al PSBD y 554 no lo siguieron.

El Equipo de Salud Bucodental lo constituyen un Odontólogo de Área y una Higienista Dental como persona de apoyo.

La aplicación de los selladores se realizaba en los gabinetes odontológicos de los Centros de Salud de las dos Zonas Básicas, una urbana y otra rural. Se empleó ácido ortofosfórico Ultra etch de Ultradent, al 38\% como grabador, rollos de algodón, como aislante, espejo , sonda y pinzas. El sellador utilizado fue Ultra seal xt plus de Ultradent, fotopolimerizable y dispensado en jeringas con boquilla aplicadora. EL fraguado se realizó con lámpara de polimerización.

Una vez seleccionado el escolar y situado en el gabinete dental, limpiábamos fosas y fisuras del molar que iba a recibir el sellador con cepillo de profilaxis, sin pasta fluorada. Después aislábamos el diente con rollos de algodón y procedíamos al grabado de la superficie a sellar durante 20-30 segundos, lavábamos durante 20 segundos con chorro de agua y secábamos la zona con aire hasta alcanzar un color blanco-tiza.

Posteriormente aplicábamos el sellador con su jeringa, evitando burbujas y sobre-rellenado, se polimerizaba durante 20 segundos, chequeábamos la oclusión con papel de articular y ajustábamos la misma si fuera necesario, utilizando contra-ángulo y fresa de pulido.

La revisión de los dientes sellados se hacía periódicamente a intervalos regulares de 6 a 12 meses, para su control y/o reposición parcial o completa. La primera revisión se hacia a los 6 meses y si no había novedad se volvía a citar al escolar a los 12 meses para nuevo control. Si existía pérdida total o parcial se reponía el sellador.

- Los criterios a los que nos ajustamos para el seguimiento y derivación fueron ${ }^{7}$ : 
- Retención completa: el sellador cubría todo el sistema de fosas y fisuras y no se apreciaba pérdida alguna (la sonda se desliza sin dificultad por todo el sellador ).

- Retención parcial: en alguna fisura había pérdida del sellador, pero la mayor parte permanecía sellado.

- Pérdida total: no se observaba sellador en la fisura o sólo quedaban pequeños restos

Diseño metodológico. Realizábamos un estudio transversal correspondiente a los 9-10 años de edad y se analizó longitudinalmente a la población diana desde 1994 hasta el curso escolar 1999-2000.

Método estadístico. Las variables del estudio se clasificaron en Cualitativas o Categóricas en escala nominal, ordinal y variables Cuantitativas. En la estadística descriptiva utilizamos medidas de centralización (media aritmética y moda), de dispersión (desviación típica) y de forma. En la estadística diferencial se utilizó la prueba de Ji-Cuadrado y medidas de asociación, escala de medida nominal y escala de medida ordinal o método.

\section{RESULTADOS}

Durante los 6 años que duró el estudio, colocamos un total de 3.979 selladores en primeros molares permanentes a nuestra P.D. Al final del estudio tan sólo encontramos 17 caries en los primeros molares sellados.

En las tablas I, II, III y IV quedan reflejados nuestros resultados especificando $n^{\circ}$ de dientes sellados, resellados y sin reposición, distinguidos por piezas y pertenencia del escolar a zona urbana o rural.

La tabla V muestra un éxito del sellado del $88,4 \%$ en la zona urbana tras 3-4 años de seguimiento de los selladores aplicados.

En la tabla VI se aprecia que la pérdida mayor del sellador en la zona urbana se produjo a los 3 años de su aplicación (58,24\% ). En relación con la zona rural podemos observar en la tabla VII un porcentaje de resellado del $7,26 \%$.

\begin{tabular}{|} 
TABLA I.- NÚMERO DE DIENTES DE LOS ESCOLARES DE LA ZONA URBANA SELLADOS, \\
RESELLADOS Y SIN REPOSICIÓN, EXPRESADOS EN PORCENTAJES, DISTINGUIDOS POR PIEZAS. \\
\begin{tabular}{|c|c|c|c|c|c|c|}
\hline DIENTE & $\begin{array}{c}N^{\circ} \text { DIENTES } \\
\text { SELLADOS }\end{array}$ & $\begin{array}{c}\% \\
\text { SELLADOS }\end{array}$ & $\begin{array}{c}N^{\circ} \text { DIENTES } \\
\text { RESELLADOS }\end{array}$ & $\begin{array}{c}\% \\
\text { RESELLADOS }\end{array}$ & $\begin{array}{c}N^{\circ} \text { DIENTES } \\
\text { SIN RESELADO }\end{array}$ & $\%$ ÉXITO \\
\hline 16 & 703 & 25,87 & 8 & 7,62 & 695 & 26,61 \\
\hline 26 & 685 & 25,21 & 15 & 14,28 & 670 & 25,65 \\
\hline 36 & 667 & 24,55 & 42 & 40 & 625 & 23,92 \\
\hline 46 & 662 & 24,36 & 40 & 38,09 & 622 & 23,81 \\
\hline TOTAL & 2717 & 100,00 & 105 & 100 & 2612 & 100 \\
\hline & & & & 3.86 & & 96.14 \\
\hline
\end{tabular}
\end{tabular}

\section{TABLA II.- NÚMERO DE DIENTES DE LOS ESCOLARES DE LA ZONA URBANA RESELLADOS DISTINGUIDOS POR EL NÚMERO DE AÑOS TRANSCURRIDOS HASTA LA REPOSICIÓN Y POR PIEZAS.}

\begin{tabular}{|c|c|c|c|c|c|c|}
\hline $\begin{array}{c}\text { AÑ̃OS } \\
\text { TRANSCURRIDOS }\end{array}$ & $\begin{array}{c}\text { DIENTE 16 } \\
\text { (NÚMERO) }\end{array}$ & $\begin{array}{c}\text { DIENTE 26 } \\
\text { (NÚMERO) }\end{array}$ & $\begin{array}{c}\text { DIENTE 36 } \\
\text { (NÚMERO) }\end{array}$ & $\begin{array}{c}\text { DIENTE 46 } \\
\text { (NÚMERO) }\end{array}$ & $\begin{array}{c}\text { TOTAL DE } \\
\text { DIENTES }\end{array}$ & $\%$ \\
\hline Antes del año & 1 & 0 & 1 & 0 & 2 & 1.9 \\
\hline $\mathrm{Al}$ año & 1 & 1 & 3 & 4 & 9 & 8.57 \\
\hline $\mathrm{A} \operatorname{los}$ 2 años & 1 & 6 & 15 & 14 & 36 & 34.28 \\
\hline $\mathrm{A} \operatorname{los} 3$ años & 5 & 8 & 21 & 19 & 53 & 50.48 \\
\hline $\mathrm{A} \operatorname{los} 4$ años & 0 & 0 & 2 & 3 & 5 & 4.76 \\
\hline TOTAL & 8 & 15 & 42 & 40 & 105 & 100.0 \\
\hline
\end{tabular}


TABLA III.- NÚMERO DE DIENTES DE LOS ESCOLARES DE LA ZONA RURAL SELLADOS, RESELLADOS, Y SIN REPOSICIÓN, EXPRESADOS EN PORCENTAJES, DISTINGUIDOS POR PIEZAS.

\begin{tabular}{|c|c|c|c|c|c|c|}
\hline DIENTE & $\begin{array}{c}N^{\circ} \text { DIENTES } \\
\text { SELLADOS }\end{array}$ & $\begin{array}{c}\% \\
\text { SELLADOS }\end{array}$ & $\begin{array}{c}N^{\circ} \text { DIENTES } \\
\text { RESELLADOS }\end{array}$ & $\begin{array}{c}\% \\
\text { RESELADOS }\end{array}$ & $\begin{array}{c}N^{\circ} \text { DIENTES } \\
\text { SIN RESELLADO }\end{array}$ & $\%$ ÉXITO \\
\hline Diente 16 & 320 & 25,36 & 7 & 9,09 & 313 & 26,41 \\
\hline Diente 26 & 320 & 25,36 & 13 & 16,88 & 307 & 25,9 \\
\hline Diente 36 & 310 & 24,56 & 36 & 46,75 & 274 & 23,12 \\
\hline Diente 46 & 312 & 24,72 & 21 & 27,27 & 291 & 24,56 \\
\hline TOTAL & 1262 & 100,00 & 77 & 100 & 1185 & 100 \\
\hline & & & & 6.10 & & 93.89 \\
\hline
\end{tabular}

TABLA IV.- NÚMERO DE DIENTES DE LOS ESCOLARES DE LA ZONA DE RURAL RESELLADOS DISTINGUIDOS POR EL NÚMERO DE AÑOS TRANSCURRIDOS HASTA LA REPOSICIÓN Y POR PIEZAS.

\begin{tabular}{|c|c|c|c|c|c|c|}
\hline $\begin{array}{c}\text { AÑOS } \\
\text { TRANSCURRIDOS }\end{array}$ & $\begin{array}{c}\text { DIENTE 16 } \\
\text { (NÚMERO) }\end{array}$ & $\begin{array}{c}\text { DIENTE 26 } \\
\text { (NÚMERO) }\end{array}$ & $\begin{array}{c}\text { DIENTE 36 } \\
\text { (NÚMERO) }\end{array}$ & $\begin{array}{c}\text { DIENTE 46 } \\
\text { (NÚMERO) }\end{array}$ & $\begin{array}{c}\text { TOTAL DE } \\
\text { DIENTES }\end{array}$ & $\%$ \\
\hline Antes del año & 0 & 1 & 0 & 0 & 1 & 1.30 \\
\hline Al año & 1 & 6 & 4 & 3 & 14 & 18.18 \\
\hline A los 2 años & 4 & 3 & 22 & 14 & 43 & 55.84 \\
\hline A los 3 años & 2 & 3 & 10 & 4 & 19 & 24.68 \\
\hline A los 4 años & 0 & 0 & 0 & 0 & 0 & 0 \\
\hline TOTAL & 7 & 13 & 36 & 21 & 77 & 100 \\
\hline
\end{tabular}

TABLA V.- NÚMERO DE DIENTES DE LOS ESCOLARES DE LA ZONA URBANA SELLADOS, RESELLADOS Y SIN REPOSICIÓN, EXPRESADOS EN PORCENTAJES, DISTINGUIDOS POR PIEZAS (AÑOS 1994 Y 1995). SEGUIMIENTO HASTA 1999.

\begin{tabular}{|c|c|c|c|c|c|c|}
\hline DIENTE & $\begin{array}{c}N^{\circ} \text { DIENTES } \\
\text { SELLADOS }\end{array}$ & $\begin{array}{c}\% \\
\text { SELLADOS }\end{array}$ & $\begin{array}{c}N^{\circ} \text { DIENTES } \\
\text { RESELLADOS }\end{array}$ & $\begin{array}{c}\% \\
\text { RESELADOS }\end{array}$ & $\begin{array}{c}\text { N}^{\circ} \text { DIENTES } \\
\text { SIN RESELADO }\end{array}$ & $\%$ ÉXITO \\
\hline Diente 16 & 203 & 25,85 & 8 & 8,8 & 195 & 28,09 \\
\hline Diente 26 & 195 & 24,84 & 13 & 14,28 & 182 & 26,22 \\
\hline Diente 36 & 194 & 24,71 & 37 & 40,65 & 157 & 22,62 \\
\hline Diente 46 & 193 & 24,58 & 33 & 36,26 & 160 & 23,05 \\
\hline TOTAL & 785 & 100,00 & 91 & 100 & 694 & 100 \\
\hline & & & & 11.6 & & $\mathbf{8 8 . 4}$ \\
\hline
\end{tabular}

TABLA VI - NÚMERO DE DIENTES DE LOS ESCOLARES DE LA ZONA URBANA RESELLADOS DISTINGUIDOS POR EL NÚMERO DE AÑOS TRANSCURRIDOS HASTA LA REPOSICIÓN Y POR PIEZAS (AÑOS 1994 Y 1995). SEGUIMIENTO HASTA 1999.

\begin{tabular}{|c|c|c|c|c|c|c|}
\hline $\begin{array}{c}\text { AÑOS } \\
\text { TRANSCURRIDOS }\end{array}$ & $\begin{array}{c}\text { DIENTE 16 } \\
\text { (NÚMERO) }\end{array}$ & $\begin{array}{c}\text { DIENTE 26 } \\
\text { (NÚMERO) }\end{array}$ & $\begin{array}{c}\text { DIENTE 36 } \\
\text { (NÚMERO) }\end{array}$ & $\begin{array}{c}\text { DIENTE 46 } \\
\text { (NÚMERO) }\end{array}$ & $\begin{array}{c}\text { TOTAL DE } \\
\text { DIENTES }\end{array}$ & $\%$ \\
\hline Antes del año & 1 & 0 & 1 & 0 & 2 & 2.2 \\
\hline Al año & 1 & 1 & 2 & 1 & 5 & 5.49 \\
\hline A los 2 años & 1 & 4 & 11 & 10 & 26 & 28.57 \\
\hline A los 3 años & 5 & 8 & 21 & 19 & 53 & 58.24 \\
\hline A los 4 años & 0 & 0 & 2 & 3 & 5 & 5.49 \\
\hline TOTAL & 8 & 13 & 37 & 33 & 91 & 100 \\
\hline
\end{tabular}




\begin{tabular}{|c|c|c|c|c|c|c|}
\hline $\begin{array}{r}\text { TABLA VI } \\
\text { RESELLAI }\end{array}$ & $\begin{array}{l}\text { IMERO DE } \\
\text { Y SIN REF } \\
\text { PIEZAS }\end{array}$ & $\begin{array}{l}\text { ENTES DE } \\
\text { ICIÓN, EX } \\
\text { OS } 1994 \text { Y }\end{array}$ & $\begin{array}{l}\text { OS ESCOLA } \\
\text { 2ESADOS EI } \\
995) . \text { SEGUII }\end{array}$ & $\begin{array}{l}\text { ES DE LA ZC } \\
\text { PORCENTAJ } \\
\text { IENTO HAST }\end{array}$ & $\begin{array}{l}\text { NA RUIRAL SEI } \\
\text { ES, DISTINGUII } \\
\text { A } 1999 .\end{array}$ & $\begin{array}{l}\text { ADOS, } \\
\text { OS POR }\end{array}$ \\
\hline DIENTE & $\begin{array}{l}N^{\circ} \text { DIENTES } \\
\text { SELADOS }\end{array}$ & $\begin{array}{c}\% \\
\text { SELLADOS }\end{array}$ & $\begin{array}{c}N^{\circ} \text { DIENTES } \\
\text { RESELADOS }\end{array}$ & $\begin{array}{c}\% \\
\text { RESELADOS }\end{array}$ & \begin{tabular}{c|}
$N^{\circ}$ DIENTES \\
SIN RESELLADO
\end{tabular} & \% ÉXITO \\
\hline Diente 16 & 102 & 25,56 & 5 & 17,24 & 97 & 26,22 \\
\hline Diente 26 & 101 & 25,31 & 8 & 27,58 & 93 & 25,13 \\
\hline Diente 36 & 99 & 24,81 & 13 & 44,83 & 86 & 23,24 \\
\hline Diente 46 & 97 & 24,31 & 3 & 10,34 & 94 & 25,40 \\
\hline \multirow[t]{2}{*}{ TOTAL } & 399 & 100,00 & 29 & 100 & 370 & 100 \\
\hline & & & & 7.26 & & 92.7 \\
\hline
\end{tabular}

\section{TABLA VIII.- NÚMERO DE DIENTES DE LOS ESCOLARES DE LA ZONA RURAL RESELLADOS DISTINGUIDOS POR EL NÚMERO DE AÑOS TRANSCURRIDOS HASTA LA REPOSICIÓN Y POR PIEZAS (AÑOS 1994 Y 1995). SEGUIMIENTO HASTA 1999.}

\begin{tabular}{|c|c|c|c|c|c|c|}
\hline $\begin{array}{c}\text { AÑOS } \\
\text { TRANSCURRIDOS }\end{array}$ & $\begin{array}{c}\text { DIENTE 16 } \\
\text { (NÚMERO) }\end{array}$ & $\begin{array}{c}\text { DIENTE 26 } \\
\text { (NÚMERO) }\end{array}$ & $\begin{array}{c}\text { DIENTE 36 } \\
\text { (NÚMERO) }\end{array}$ & $\begin{array}{c}\text { DIENTE 46 } \\
\text { (NÚMERO) }\end{array}$ & $\begin{array}{c}\text { TOTAL DE } \\
\text { DIENTES }\end{array}$ & $\%$ \\
\hline Antes del año & 0 & 0 & 0 & 0 & 0 & 0 \\
\hline $\mathrm{Al}$ año & 0 & 0 & 1 & 1 & 2 & 6.9 \\
\hline $\mathrm{A} \operatorname{los}$ años & 3 & 2 & 4 & 0 & 9 & 31.03 \\
\hline $\mathrm{A} \operatorname{los} 3$ años & 2 & 3 & 8 & 2 & 15 & 51.72 \\
\hline $\mathrm{A} \operatorname{los} 4$ años & 0 & 3 & 0 & 0 & 3 & 10.34 \\
\hline TOTAL & 5 & 8 & 13 & 3 & 29 & 100 \\
\hline
\end{tabular}

Antes del año vemos, en la tabla VIII, como en la zona rural no hubo pérdida alguna de selladores en el periodo transcurrido desde el curso escolar 199495 hasta 1999.

\section{DISCUSIÓN}

Wendt y Goran ${ }^{8}$ en el año 1988 , evaluaron la retención de selladores · A los 8 años el $80 \%$ de fisuras selladas mostraron una retención total del sellador, al año la retención completa la mostraban el $98 \%$ de los selladores.

Amid et $\mathrm{al}^{9}$ en 1989 , constataron que el $87 \%$ de los selladores se mantenían retenidos al año, a los dos años el $83 \%$ y a los tres años el $79 \%$. Nosotros en nuestro estudio encontramos que el mayor porcentaje de pérdidas de selladores en las Zonas Básicas de Salud se presentó, de forma mayoritaria, a los 2 y 3 años de su aplicación, más a los 3 . Estamos de acuerdo con algunos autores ${ }^{10}$ en afirmar que el porcentaje de retención al año suele estar en la mayoría de los estudios por encima del $80 \%$, disminuyendo en años sucesivos. Estos mismos autores ${ }^{10}$ encontraron una retención de selladores a los tres años del $91,5 \%$ al $95,3 \%$. Otros autores ${ }^{11}$ publican cifras de retención de los selladores a los tres años del 94\%; estas cifras las podemos comparar con las obtenidas por nosotros en la población rural a los 3-4 años de la aplicación del sellador $(92,74 \%)$.

Existen numerosos estudios ${ }^{12}$ que no presentan resultados homogéneos (oscilaban entre el $28 \%$ y el $100 \%$ ) en cuanto a la retención de selladores. Las diferencias podrían explicarse por la realización técnica de los mismos. En algunos se realizaron bajo condiciones clínicas óptimas, mientras que en otros se realizaron bajo condiciones de campo más deficientes, llegando a ser colocados por personal de diferente capacitación profesional con o sin ayudante.

Nosotros, como ya hemos explicado, no usamos dique de goma como forma de aislamiento, ya que no es necesario (especialmente en salud pública oral) si se maneja correctamente la técnica del aislamien- 
to relativo. Se han obtenido los mismos resultados en cuanto a la utilización de rollos de algodón o dique de goma en la aplicación de selladores. Utilizando correctamente el aislamiento relativo se han obtenido porcentajes de retención muy altos en la literatura ${ }^{13}$. Además el gran inconveniente del dique de goma es que frecuentemente requiere una aplicación previa de anestesia tópica en el lugar donde se coloca la grapa e incluso punción anestésica.

Nuestros resultados coinciden en lo argumentado por numerosos autores: cuanto mayor adiestrado sea el profesional y cuanto más conozca la técnica, los porcentajes de retención van a aumentar ${ }^{14}$.

En cuanto al tipo de sellador (auto o fotopolimerizable) hay numerosos estudios ${ }^{10,15}$ que comparan las diferencias en su efectividad, obteniendo mejores resultados con los autopolimerizables. Sin embargo, hay que tener en cuenta que en muchos de estos trabajos, los selladores fotopolimerizables eran los de primera generación (tenían problemas con la luz ultravioleta), los cuales no tenían la misma calidad que los dispensados actualmente. Nosotros pensamos que los dos tipos de selladores son eficaces si se utilizan correctamente, aunque quizás sea más cómodo y rápido manipular los fotopolimerizables.

\section{OBJETIVOS}

Llodrá y colaboradores ${ }^{15}$, en 1993 , en su estudio hecho sobre los factores que tenían influencia en la efectividad de los selladores, consideraban que en periodos iguales de seguimiento, no se hallaban diferencias significativas en la efectividad entre las diferentes marcas de selladores.

Los estudios clínicos que han utilizado selladores de tercera generación han sido recogidos por Ripa ${ }^{16}$ en 1993; ofrecieron una retención total a los 12 meses que oscilaba entre el $75 \%$ y $97 \%$, a los 24 meses del $60 \%$ al $98 \%$ y a los 36 meses del $43 \%$ al $83 \%$. La retención de selladores a los 5 años fue del $48 \%$.

Si comparamos la cifra de 36 meses obtenidas por Ripa $^{16}$ con las obtenidas por nosotros a los 3-4 años, nuestras cifras las podemos situar en un nivel más alto.
Bravo et $\mathrm{al}^{17}$ a los 12 meses en su estudio obtuvo una retención del $87,3 \%$, del $74,7 \%$ a los 24 meses y del $61,7 \%$ a los 36 meses, también utilizando selladores de tercera generación, al igual que nosotros.

Si la pérdida temprana de selladores se debe a una mala realización de la técnica. ${ }^{18}$ ¿Cuáles son los motivos responsables de una pérdida tardía?. Lo que realmente puede influir en una pérdida futura es el desgaste del mismo sellador, influido a la vez por las fuerzas de la oclusión, fuerzas de cizalla y filtración marginal. Algunos autores ${ }^{6}$ propugnan que un sellador bien colocado debería durar entre 3 y 7 años.

Algo muy positivo que realizábamos a nuestros escolares, era la revisión anual o semestral, que entre otros motivos servía para volver a aplicar el sellador perdido y así mantener la integridad del mismo para a la vez prevenir la caries dental. Cuando veíamos una pérdida parcial resellábamos, ya que como argumentan algunos autores, ${ }^{13}$ la efectividad es del $100 \%$ cuando el sellador se coloca periódicamente, si es que sufre algún tipo de pérdida ya sea total o parcial.

Coincidimos con algunos autores, ${ }^{19}$ en decir que la efectividad se debe sobre todo a las revisiones realizadas y no al tipo de sellador. Es necesario reponer cuando haya pérdidas, ya que está demostrado que no se desarrolla ninguna lesión cariosa en una superficie completamente sellada.

Podemos influir de algún modo en la rentabilidad del sellador; la colocación de muchos selladores en una única cita, reduce el coste por sellado ${ }^{20,21}$. Es este aspecto el que siempre hemos tenido presente en la realización de nuestro estudio; cuando un niño necesitaba selladores en los cuatro molares, éstos se les aplicaban en una sola sesión.

Refiriéndonos a los primeros molares permanentes, que más frecuentemente no sufrieron pérdidas del sellador, nos encontramos que el molar 16, en Valdepeñas, obtuvo el mayor éxito de sellado seguido del 26, 46 y 36. En la Zona Básica de Salud de Villanueva de los Infantes, el porcentaje mayor de éxito lo obtuvo el 16 seguido del 26, 46 y 36.

La mayoría de los estudios demuestran, que los molares del maxilar inferior presentan menos reten- 
ción ${ }^{1}$. Esto podría explicarse por los problemas con el acceso y el control de la saliva ${ }^{21}$.

\section{CONCLUSIONES}

$1^{\circ}$ Los selladores preservan la estructura del diente, son poco traumáticos para el paciente pediátrico, de reducido coste económico y suponen un ahorro de tiempo total tanto para el paciente como para el operador.

$2^{\circ}$ La técnica de sellado ha sido correcta y eficaz para el fin perseguido.

$3^{\circ} \mathrm{El}$ porcentaje de éxito obtenido en la zona rural ha sido mayor que el obtenido en la zona urbana.

$4^{\circ}$ La perdida del sellador se produce con más frecuencia a los tres años de su aplicación.

$5^{\circ}$ No hemos encontrado relación entre pérdida de sellador y molar sellado.

\section{BIBLIOGRAFÍA}

1. Pankala K, Kentala J, Laippala P, Mattila K. Changes in the oral health of adolescents threated by the finish public dental services between the ages of 13 and 15 years. Community Dent Oral Epidemiol 1998; 26: 49-154.

2. Riobóo R. La evolución de la salud buco-dental en la población europea durante la última década: Variables implicadas. Av Odontoestomatol 1998; 14(2): 119-31.

3. Van Rijkom HM, Truin GJ, Van't Hof MA. A Meta Analysis of Clinical Studies on the Caries Inhibiting Effect of Fluoride fel Treatment. Caries Res 1997; 32: 83-92.

4. Jackson Brown DDS, Thomas P, Wall MA, Vickie Lazar MA.Trends in Untreated caries in permanent teeth of children 6 to 18 years old. J Am Dent Assoc 1999 Nov; 4 (130):1637-44.

5. Birkeland JM, Haugejorden O, Ramm von der
Fehr F. Some Factors Associated with the Caries Decline among Norwegian Children and Adolescents: Age Specific and Cohort Analyses. Caries Res 2000 Marzo-Abril; 34: 109-16.

6. Deery C, Care R, Chesters R, Huntington E, Stelmachonoka S, Gudkina Y. Prevalence of dental caries in Latvian 11to 15 Years Old Children and the Enhanced Diagnostic Yield of Temporary Tooth Separation, FOTI and Electronic Caries Measurement. Caries Res 2000; 34: 2-7.

7. Pitts NB, Evans DJ, Nugent ZJ. The denal caries experience of 14 years old children in the United Kindom. Surveys coordinated by the Brithish Association for the Study of Community Dentistry in 1998/1999. Community Dent Health 2000; 17: 48-53.

8. Wendt L, Goran K. Fissure sealant in permanent firs molars after 10 years. Swed Dent J 1988, 12: 181-5.

9. Amid I, King W, Clark C. An Evaluation of the Saskatchewan pit and Fissure Sealant Program: a longitudinal followup. J Public Health Dent 1989; 40: 206-11.

10. García JI, López P, Varón T. Evaluación de una estrategia de educación para la salud oral en el ámbito escolar: Concurso "Limpia tus dientes y gana”. Arch Odontoestomatol Prev Comunitaria 2000; 16(9): 602-11.

11. Worthington H, Kirsty B, Jeanette Mooney BA, Fiona A, Anthony S. A Cluster Randomized Controlled Trial of a Dental Health Education Program for 10 year old Children. J Public Health Dent 2001; 60 (1): 22-7.

12. Sánchez M. Dimensiones educativas y sanitarias de la educación para la salud en la escuela: una experiencia participativa y global en dos zonas de salud de Murcia. Rev Sanid Hig Publica 1993; 67: 293-304.

13. Jackson Brown DDS, Thomas P, Wall MA, Vickie Lazar MA.Trends in total caries experience: permanent and primary teeth. J Am Dent Assoc 2000 Feb; 131: 223-31. 
14. Rayner JA. A dental health education programme including home visits for nursery. $\mathrm{Br}$ Dent $\mathrm{J}$ 1992; 172: 57-62.

15. Llodra JC, Bravo M, Delgado Rodríguez M, Baca P, Gálvez R. Factors influencing the effectiveness of sealants a meta analysis. Community Dent Oral Epidemiol 1993; 21: 261-8.

16. Ripa LW. Sealants revisited: an update of the effectiveness of pit and fissure sealants. CariesRes 1993; 27 (1): 77-82.

17. Bravo M, Llodra JC, Baca P, Osorio E Effectiveness of visible light fissure sealant (Delton) versus fluoride barniz (Duraphat) 24 month clinical trial. Community Dent Oral Epidemiol 1996; 24: 42-6.

18. Stewart JE, Jacobs-Schoen M, Padilla MR, Wolfe GR, Hartz GW. The effect of acognitive behavioral intervention on oral higyene. J Clin Periodontol 1991; 18: 219-22.
19. Bravo M, Llodra JC, Baca P, Osorio E, Junco P. Selladores de fisuras frente a barniz de flúor en primeros molares permanentes: evaluación económica. Aten Primaria 1995; 15 (3): 143-7.

20. Brearley L, Calache $H$, Morgan $M$. The retention of pit and fissure sealants placed in primary schoolchildren by Dental Health Services, Victoria. Aust Dent J 1994; 42 (4): 233-9.

21. Axelsson P, Rolandsson M, Bjerner B. How swedish dental hygienists apply trainning program in the field. Community Dent Oral Epidemiol 1993; 27: 37-49.

\section{CORRESPONDENCIA}

Dtra. Isabel Navarro Montes

C/Escuelas $281^{\circ} \mathrm{F}$. 13300 Valdepeñas ( Ciudad Real)

E-mail: Lpesoo@infomed-dental.com 